TRANSACTIONS OF THE

AMERICAN MATHEMATICAL SOCIETY

Volume 361, Number 8, August 2009, Pages 4027-4044

S 0002-9947(09)04562-0

Article electronically published on March 20, 2009

\title{
EXISTENCE OF TRAVELING DOMAIN SOLUTIONS FOR A TWO-DIMENSIONAL MOVING BOUNDARY PROBLEM
}

\author{
Y. S. CHOI AND ROGER LUI
}

\begin{abstract}
In this paper we prove the existence of a traveling domain solution for a two-dimensional moving boundary problem. Specifically, we prove the existence of a domain that travels to the right at a constant speed $k$ and a function $b$ which solves a porous medium type equation in the domain with constant Dirichlet boundary condition. The proof is by Schaefer's fixed point theorem. The result may be viewed as an extension of the existence of traveling cell solutions of a one-dimensional cell motility model proved by the authors and Juliet Lee (2004).
\end{abstract}

\section{INTRODUCTION}

Suppose $u$ satisfies a parabolic partial differential equation (PDE) in a bounded domain in $\mathbf{R}^{2}$ that moves in time. Let the domain be denoted by $\Omega_{t}$ at time $t$ and suppose its boundary is given by the zero level curve of a function $\psi$, i.e. $(x, y) \in \partial \Omega_{t}$ if and only if $\psi(x, y, t)=0$. Frequently, $\psi$ is chosen to be a distance function with $\Omega_{t}=\{(x, y) \mid \psi(x, y, t)<0\}$. It is well known that $\psi$ satisfies a Hamilton-Jacobi type equation $\psi_{t}+V_{n}|\nabla \psi|=0$, where $V_{n}$ is the speed of $\partial \Omega_{t}$ in the outward normal direction $\mathbf{n}$. The function $V_{n}$ depends on the properties of the domain as well as $u$ or its first derivatives. The moving boundary problem (MBP) can be stated as follows: given $V_{n}$ and $T>0$, find $u$ and the zero level curve of $\psi$ such that $u$ satisfies the parabolic PDE with prescribed boundary conditions in $\Omega_{t} \times[0, T)$ and $\psi$ satisfies the above Hamilton-Jacobi type equation in $\mathbf{R}^{2} \times[0, T)$.

The mathematical problem studied in this paper arises from extending a onedimensional cell motility problem by Mogilner and Verzi [11] to two dimensions. Details of this extension will be explained later in this section. The resulting PDE is of the form $u_{t}=\Delta u^{m}-u, m \geq 1$, with boundary condition $u=u_{0}$ on $\partial \Omega_{t}$. The question addressed here is under what conditions on $V_{n}$ does this MBP possess a traveling domain solution that moves to the right at a constant speed $k$. In particular, we look for a pair of functions $\tilde{u}(x, y), \tilde{\psi}(x, y)$ and a constant $k$ such that $u(x, y, t)=\tilde{u}(x-k t, y)$ and $\tilde{\psi}(x-k t, y)$ satisfy the MBP. Carrying out the differentiation, $\tilde{u}$ satisfies the equation $\Delta \tilde{u}^{m}+k \tilde{u}_{x}-\tilde{u}=0$ in the domain $\Omega$, and $\tilde{u}=u_{0}$ on $\partial \Omega$ where $\partial \Omega$ is the zero level curve of the function $\psi$ that satisfies the equation $-k \tilde{\psi}_{x}+V_{n}|\nabla \tilde{\psi}|=0$. If $\partial \Omega$ is smooth, then one can define the normal angle

Received by the editors December 13, 2005 and, in revised form, May 7, 2007.

2000 Mathematics Subject Classification. Primary 35R35, 92C17.

Key words and phrases. Cell motility, moving boundary problem, traveling domain solutions.

The first author's research was partially supported by NIH grant no. 5P41-RR013186-07.

The second author's research was partially supported by NSF grant no. DMS-0456570.

(C)2009 American Mathematical Society 
$\phi$ on the boundary as the angle between $\mathbf{n}$ and $\mathbf{i}=(1,0)$. Then $\mathbf{n}=(\cos \phi, \sin \phi)=$ $\nabla \tilde{\psi} /|\nabla \tilde{\psi}|$ since $\tilde{\psi}=0$ on $\partial \Omega$. Hence finding $\tilde{\psi}$ is equivalent to finding a domain $\Omega$ that satisfies the relation $k \cos \phi=V_{n}$ on the boundary.

The purpose of this paper is to prove the existence of a traveling domain solution of the above MBP when $V_{n}$ has the form given by the right hand side of (1.1) (c) below. The precise result is formulated in the following theorem.

Theorem 1.1. Let $m \geq 1$ and let $V_{d}, b_{0}$ be positive. There exists a $C^{2}$ convex domain $\Omega \subset \mathbf{R}^{2}$ symmetric about the $x$-axis and centered at the origin, a positive function $b \in C^{2, \alpha}(\bar{\Omega})$ and a constant $k>0$, such that

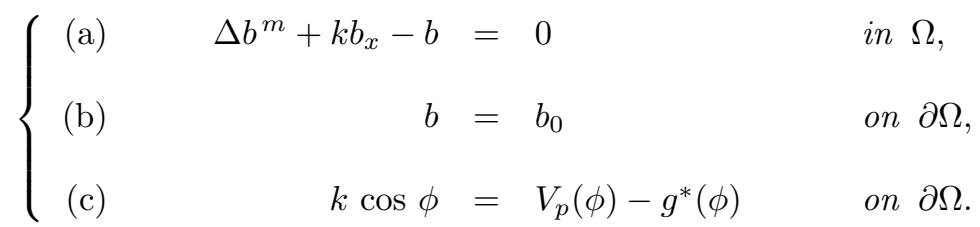

In (1.1) (c), $g^{*}$ is a $C^{1}$ function defined on $[0, \pi]$ such that $0 \leq g^{*}(\phi) \leq|\nabla b|(\phi)$ on $[0, \pi]$. Its precise definition is given in Remark 1 below. Also,

$$
V_{p}(\phi)=\left(\frac{1}{s_{0}^{2}}-\frac{V_{d}}{2}\right)+\left(\frac{1}{s_{0}^{2}}+\frac{V_{d}}{2}\right) \cos \phi-\frac{d \phi}{d s},
$$

$s_{0}=\frac{1}{2}|\partial \Omega|$, and $s$ is arc-length measured counter clockwise from the point $F$ on $\partial \Omega$ (see Figure 1).

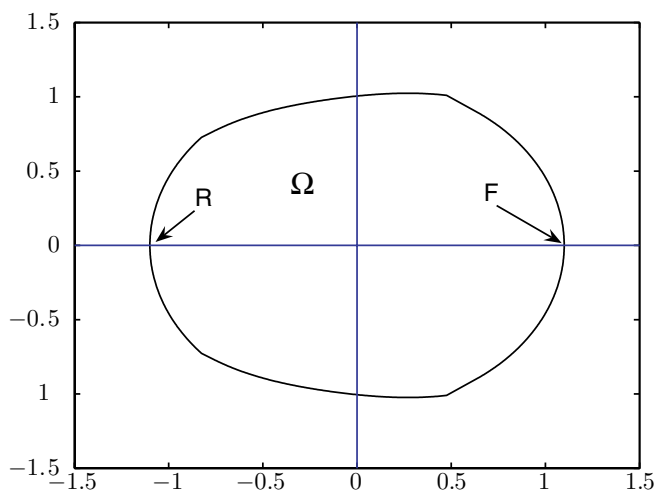

FiguRE 1. Front and rear of a domain $\Omega$.

The paper is rather technical, so we begin with some general remarks which will help the readers understand our ideas and proofs.

Remark 1. We first explain how $g^{*} \in C^{1}[0, \pi]$ is constructed from a given $g \in$ $C[0, \pi], g \geq 0$. The function $g^{*}$ should be thought of as a regularization of $g$. The reason why we need to use $g^{*}$ instead of $g(\phi)=|\nabla b|(\phi)$ in (1.1) (c) will be explained at the end of $\S 3$.

Let $\left\{I_{i}=\left(a_{i}, b_{i}\right), i=1, \ldots, N\right\}$ be a covering of the interval $[0, \pi]$ and let $\left\{\xi_{i} \in C^{\infty}(\mathbf{R}), i=1,2, \ldots, N\right\}$ be a partition of unity subordinate to $\left\{I_{i}\right\}_{i=1}^{N}$; i.e. 
$\xi_{i}$ has compact support in $I_{i}$ and $\sum_{i=1}^{N} \xi_{i}(\phi)=1$ for $\phi \in[0, \pi]$. Define

$$
g^{*}(\phi)=\sum_{i=1}^{N} \xi_{i}(\phi) \min _{\psi \in \overline{I_{i}}} g(\psi) \text {. }
$$

For any $\phi \in[0, \pi]$, let $J_{\phi}=\left\{i \in\{1, \ldots, N\} \mid \phi \in I_{i}\right\}$. Then since $\xi_{j}(\phi)=0$ if $j \notin J_{\phi}$, we have

$$
g^{*}(\phi)=\sum_{i \in J_{\phi}} \xi_{i}(\phi) \min _{\psi \in \overline{I_{i}}} g(\psi) \leq \sum_{i \in J_{\phi}} \xi_{i}(\phi) g(\phi)=g(\phi) .
$$

From (1.3), it is clear that $(\lambda g)^{*}=\lambda g^{*}$ for $0 \leq \lambda \leq 1$.

Lemma 1.1. Let $g_{n} \rightarrow g$ in $C[0, \pi]$. Then $g_{n}^{*} \rightarrow g^{*}$ in $C^{j}[0, \pi]$ for any nonnegative integer $j$.

Proof. Since the $\xi_{i}$ 's are $C^{\infty}$ functions, it suffices to show that $\min _{\psi \in \overline{I_{i}}} g_{n}(\psi) \rightarrow$ $\min _{\psi \in \overline{I_{i}}} g(\psi)$ as $n \rightarrow \infty$ for each $i=1,2, \ldots, N$. Fix $I_{i}$ and let $x_{n}, x_{0} \in \overline{I_{i}}$ be such that

$$
g_{n}\left(x_{n}\right)=\min _{\psi \in \overline{I_{i}}} g_{n}(\psi) \quad \text { and } \quad g\left(x_{0}\right)=\min _{\psi \in \overline{I_{i}}} g(\psi) .
$$

Then $g\left(x_{0}\right)-g_{n}\left(x_{n}\right) \leq g\left(x_{n}\right)-g_{n}\left(x_{n}\right) \leq\left\|g-g_{n}\right\|_{C[0, \pi]}$ and $g_{n}\left(x_{n}\right)-g\left(x_{0}\right) \leq$ $g_{n}\left(x_{0}\right)-g\left(x_{0}\right) \leq\left\|g-g_{n}\right\|_{C[0, \pi]}$. Therefore, $\left|g\left(x_{0}\right)-g_{n}\left(x_{n}\right)\right| \leq\left\|g-g_{n}\right\|_{C[0, \pi]}$. The proof of the lemma is complete.

We further remark that if $g \in C[0, \pi]$, then $g^{*} \rightarrow g$ in $C[0, \pi]$ as the maximum size of the covering $\max _{1 \leq i \leq N}\left|I_{i}\right| \rightarrow 0$. Thus the solution to our problem can be a good approximation to the traveling domain solution, if it exists, when $g^{*}$ is replaced by $g$ in (1.1) (c).

Remark 2 . With respect to the moving coordinates, $\Omega$ will appear as stationary and has the following properties: (i) $\partial \Omega \in C^{2}$, (ii) $\Omega$ is convex, (iii) $\Omega$ is symmetric about the $x$-axis, (iv) the origin bisects the line $\overline{R F}$ (see Figure 1). Therefore, the lines tangent to $\Omega$ at $F$ and $R$ must be vertical; i.e. $\phi=0$ at $F$ and $\phi=\pi$ at $R$. Note that for a $C^{2}$ domain, $d \phi / d s=\kappa$ is the curvature of its boundary and a domain is convex if and only if $\kappa \geq 0$.

Remark 3. The proof of Theorem 1.1 is via Schaefer's fixed point theorem (see Theorem 5.1). Loosely speaking, the idea is that we start with a positive continuous function $g$ defined on $[0, \pi]$ which represents a guess of $|\nabla b|(\phi)$ along the upper half of $\partial \Omega$. We define $g^{*}$ from $g$ according to Remark 1 above and use equation (1.1) (c) to recover $k$ and $\Omega$. This step is denoted by the map $T_{1}$. Next we solve (1.1) (a) and (b) with the $k$ and $\Omega$ just found and obtain $|\nabla b|(s)$. If $\Omega$ is strictly convex, then we can identify $s$ with $\phi$ and define $\tilde{g}(\phi)=|\nabla b|(s(\phi))$. This step is denoted by the map $T_{2}$. Let $T=T_{2} \circ T_{1}$. We then show that $T$ has a fixed point which is then the traveling domain solution to our MBP.

We now explain the connection between our 2D model and the 1D cell motility model of Mogilner and Verzi. In [11, Mogilner and Verzi proposed a model to describe the one-dimensional crawling motion of a nematode sperm cell. They assumed that the cytoskeletal filaments inside the cell are bundled together near the front of the cell to push the front forward with a speed $V_{p}$ and depolymerize near the rear to pull the cell body forward with a speed $V_{d}$. Moreover the elasticity 
of the bundled filaments will generate contractile forces throughout the cell. Let the length density of the bundled filaments be denoted by $b$. Then it is implied by the Mogilner and Verzi model that $b_{x x}^{2}+k b_{x}-b=0$ is the governing traveling domain equation in 1D, where $k$ is the traveling speed. For a 2D model, $\sigma=\left(\sigma_{i j}\right)$ is a tensor $(2 \times 2$ symmetric matrix $)$. Suppose we assume that stress is isotropic so that $\sigma=\theta I$, where $I$ is the identity matrix and the scalar function $\theta$ depends on the densities of various proteins inside the cell. Then the traveling domain equation (1.1) (a) is a simple generalization of the 1D case. Similarly the boundary conditions can be generalized. In particular zero stress on the cell boundary leads to $b=b_{0}$ and the normal speed at the boundary is given by $V_{n}=V_{p}-|\nabla b|$, where $|\nabla b|$ is the retraction speed toward the cell center due to the contractile force of the filaments. In the formula (1.2) for $V_{p}$, we have added the term $-d \phi / d s$ which represents membrane tension. Therefore, the equations we study in this paper may be considered as a 2D cell motility model assuming a special form of the stress tensor.

We close this section with some references. The literature on traveling wave solutions (see [14, 9]) and moving boundary problems is huge, but we are unable to find any work similar to what we did in this paper. For example in 3 Brazhnik and Tyson studied the existence of traveling wave solutions of Fisher's equation in $\mathbf{R}^{2}$ and in 22 Berstycki and Nirenberg gave a complete study of the existence of traveling front solutions of the equation $u_{t}=\Delta u-\alpha(y) u_{x}+f(u)$ in a cylinder in

$\mathbf{R} \times \omega$ where $\omega$ is a bounded smooth domain in $\mathbf{R}^{n-1}$. However the domains are fixed in both papers. On the other hand, what we called moving boundary problems often come under different names depending on the applications (e.g. gas bubble rising in a liquid) but almost all of them are two-phase flow, and traveling waves may not exist or may be called something else in these applications. A good example is the existence of finger solutions for the Hele-Shaw equation [13]. There, the domain is a strip $\Omega=\{(x, y) \mid-\infty<x<\infty,-L<y<L\}$, and there is a free interface in this domain that separates two immiscible fluids. The fluid pressure satisfies the Laplace equation in $\Omega$ except on the free interface, and boundary and jump conditions that are related to the curvature are imposed on the interface. There are also many other areas of applications such as flame propagation, dendrites, etc. The book by Pierce Pelcé [12] is a good source of these types of problems. In the case of 1D, our earlier papers (see [4, [5], 6]) established the well posedness of both the MBP and the traveling domain problem. The linearized stability of the traveling domain solution has also been studied in 7 .

The organization of this paper is as follows. In $\S 2$, we show how to construct the domain $\Omega$ using equation (1.1) (c). Recognizing the form of $V_{n}$ and using (1.1)(c) to recover the domain are the key ideas in this paper. In $\S 3$ and $\S 4$, we describe the constructions of the maps $T_{1}$ and $T_{2}$, respectively. In $\S 5$, we prove Theorem 1.1. For the rest of the paper, we assume that $m \geq 1, V_{d}$ and $b_{0}$ are given positive constants.

\section{Construction of the domain $\Omega$}

The following lemma defines two constants $m_{1}$ and $m_{2}$ which depend only on $m$, $V_{d}$ and $b_{0}$. 
Lemma 2.1. Let $m_{2}, m_{1}$ be the unique positive roots of the equations $2 / x-V_{d} x-$ $2 \pi=0$ and $2 / x-V_{d} x-2 \pi-x^{2} /\left(m b_{0}^{m-2} \pi\right)=0$, respectively. Then $m_{1}<m_{2}$ and $2 / m_{1}^{2}>2 / m_{2}^{2}>V_{d}$.

Proof. Clearly $m_{2}=\left(-\pi+\sqrt{\pi^{2}+2 V_{d}}\right) / V_{d}$. Let $q(x)=2 / x-V_{d} x-2 \pi-x^{2} /\left(m b_{0}^{m-2} \pi\right)$. Then $q^{\prime}(x)<0$ for $x>0, q \rightarrow \infty$ as $x \rightarrow 0$ and $q \rightarrow-\infty$ as $x \rightarrow \infty$. Therefore, $q$ has a unique positive root $m_{1}$. Since $q\left(m_{2}\right)=-m_{2}^{2} /\left(m b_{0}^{m-2} \pi\right)<0$, it is clear that $m_{1}<m_{2}$ and

$$
\frac{2}{m_{1}^{2}}>\frac{2}{m_{2}^{2}}=\frac{1}{2}\left(\sqrt{\pi^{2}+2 V_{d}}+\pi\right)^{2}>V_{d} .
$$

The proof of the lemma is complete.

The next proposition shows that if we are given the normal angle $\phi$ as a function of arc-length, then we can reconstruct the domain $\Omega$. In the lemma, $s_{0}$ is half the perimeter of $\Omega$.

Proposition 2.1. Let $0 \leq \phi(s) \leq \pi$ belong to $C^{1}\left[0, s_{0}\right]$ and suppose $\phi^{\prime}>0$ on $\left[0, s_{0}\right)$. Let $(x(s), y(s))$ satisfy the equations

$$
\left\{\begin{aligned}
\text { (a) } & \frac{d x}{d s}=-\sin \phi(s), \\
\text { (b) } & \frac{d y}{d s}=\cos \phi(s)
\end{aligned}\right.
$$

for $0 \leq s \leq s_{0}$. Then $\phi(s)$ is the normal angle of the curve $(x(s), y(s)), 0 \leq s \leq s_{0}$, where $s$ is the arc-length. If in addition, $y(0)=0, y\left(s_{0}\right)=0, \phi(0)=0$, and $\phi\left(s_{0}\right)=$ $\pi$, then $(x(s), y(s)), 0 \leq s \leq s_{0}$, is the top half of a domain $\Omega$ symmetric about the $x$-axis with $C^{2}$ boundary. By choosing $x(0)=\frac{1}{2} \int_{0}^{s_{0}} \sin \phi(\xi) d \xi$, the origin bisects the line obtained from the intersection of $\Omega$ and the $x$-axis.

Proof. For a parameterized curve $(x(s), y(s))$, the tangent direction is given by $(d x / d s, d y / d s)$. Since $(d x / d s, d y / d s) \cdot(\cos \phi, \sin \phi)=0$ and $0 \leq \phi \leq \pi$ so that $\sin \phi>0, \phi$ is the normal angle. Since $\sqrt{(d x / d s)^{2}+(d y / d s)^{2}}=1, s$ is the arclength.

Now suppose $y(0)=0, y\left(s_{0}\right)=0, \phi(0)=0$ and $\phi\left(s_{0}\right)=\pi$. From our assumptions on $\phi$ and equation (2.5) (b), $y$ has a unique local maximum and $y(s)>0$ on $\left(0, s_{0}\right)$. By reflecting the region bounded between the curve $(x(s), y(s))$ and the $x$-axis about the $x$-axis, we obtain a domain $\Omega$. This is equivalent to extending $y$ and $\phi$ as odd functions and $x$ as an even function on $\left[-s_{0}, s_{0}\right]$, and (2.5) is clearly still valid on $\left[-s_{0}, s_{0}\right]$. Moreover, $\phi \in C^{1}\left[-s_{0}, s_{0}\right]$ and $x, y \in C^{2}\left[-s_{0}, s_{0}\right]$. It is easy to check that $\Omega$ is a $C^{2}$ domain, in particular $\left(x\left(s_{0}\right), 0\right)=\left(x\left(-s_{0}\right), 0\right)$. The last statement in this proposition follows by imposing the condition $x(0)+x\left(s_{0}\right)=0$. The proof of the lemma is complete.

Consider equation (1.1) (c). For convenience, let $A=1 / s_{0}^{2}-V_{d} / 2$ and $B=$ $A+V_{d}$. Equation (1.1) (c) is a differential equation in $\phi$ with two unknowns $s_{0}$ and $k$, so the boundary conditions $\phi(0)=0, \phi\left(s_{0}\right)=\pi$ are not enough to determine them. Therefore, we include equation (2.5) (b) with boundary conditions $y(0)=$ $0, y\left(s_{0}\right)=0$. This system together with the above 4 boundary conditions will help us determine $s_{0}$ and $k$. We then use Proposition 2.1 to recover the domain $\Omega$. 
It is more convenient to study the differential equations on a fixed interval; we therefore map $\left[0, s_{0}\right]$ onto $[0,1]$ by the transformation $t=s / s_{0}$. The boundary value problem is

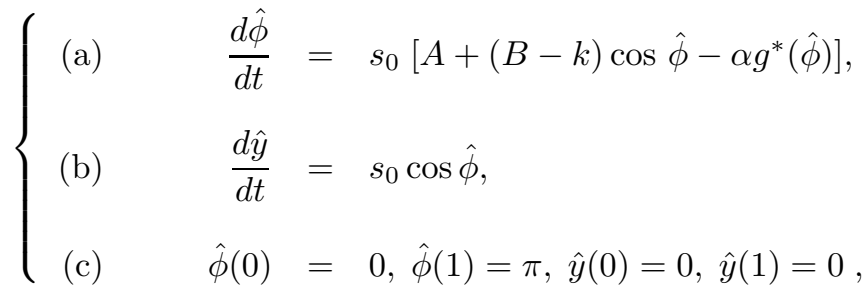

where $\hat{\phi}(t)=\phi\left(s_{0} t\right)$ and $\hat{y}(t)=y\left(s_{0} t\right)$. Note the addition of $\alpha$ in front of $g^{*}(\hat{\phi})$ in (2.6) (a).

Proposition 2.2. Let $g^{*} \in C^{1}[0, \pi]$ be a nonnegative function with $\left\|g^{*}\right\|_{C[0, \pi]} \leq$ $M_{1}$. Let $m_{1}^{*}=\left(-\pi+\sqrt{\pi^{2}+2 V_{d}+4 M_{1}}\right) /\left(V_{d}+2 M_{1}\right)$. Then for $0 \leq \alpha \leq 1$, (2.6) has a unique solution $\left(s_{0}, k, \hat{\phi}, \hat{y}\right)$, where $\hat{\phi} \in C^{2}[0,1], \hat{y} \in C^{3}[0,1], d \hat{\phi} / d t>0$, and

$$
\text { (a) } m_{1}^{*} \leq s_{0} \leq m_{2}, \quad \text { (b) } V_{d} \leq k \leq \frac{2}{m_{1}^{* 2}}, \quad \text { (c) } \frac{d \hat{\phi}}{d t} \leq \frac{4 m_{2}}{m_{1}^{* 2}} \text {. }
$$

Proof. The proof is rather technical and is given in Appendix A. We first show that the results are true when $\alpha=0$ and then use degree theory to show that they are true for all $\alpha \in[0,1]$.

For the rest of this paper, we shall let $X=C[0, \pi]$ and $X^{+}=\{g \in X \mid g \geq 0\}$. Also, we only use Proposition 2.2 with $\alpha=1$ in (2.6) (a), and $\alpha$ will be used later to denote a Hölder exponent.

\section{The MaP $T_{1}$}

We are now ready to define the map $T_{1}$. Let $g \in X^{+}$. Define $g^{*} \in C^{1}[0, \pi]$ according to Remark 1 in $\S 1$ and let $\left(s_{0}, k, \hat{\phi}, \hat{y}\right)$ be the unique solution of (2.6) according to Proposition 2.2. Let $T_{1}(g)=\left(s_{0}, k, \hat{\phi}, \hat{y}\right)$. The bounds in (2.7) depend on $m_{1}^{*}$, which in turn depends on $\|g\|_{X}$. From Proposition 2.1, we can construct a $C^{2}$ domain $\Omega$ with the properties listed in Remark 2. In what follows, we shall frequently write $T_{1}(g)=(k, \Omega)$ instead of $\left(s_{0}, k, \hat{\phi}, \hat{y}\right)$. This should not cause any confusion.

Lemma 3.1. The map $T_{1}: X^{+} \rightarrow Y=\mathbf{R} \times \mathbf{R} \times C[0,1] \times C^{1}[0,1]$ is continuous and compact. Let $T_{1}(g)=\left(s_{0}, k, \hat{\phi}, \hat{y}\right)$ for any $g \in X^{+}$. Then $\hat{\phi}$ and $\hat{y}$ satisfy all the hypotheses in Proposition 2.1 to construct a $C^{2}$ domain centered at the origin and symmetric about the $x$-axis.

Proof. Let $g_{n} \rightarrow \bar{g}$ in $X^{+}$. Then there exists $M_{1}>0$ such that $\left\|g_{n}^{*}\right\|_{X} \leq\left\|g_{n}\right\|_{X} \leq$ $M_{1}$ and $\left\|\bar{g}^{*}\right\|_{X} \leq\|\bar{g}\|_{X} \leq M_{1}$ for all $n$. Let $T_{1}\left(g_{n}\right)=\left(k_{n},\left(s_{0}\right)_{n}, \hat{\phi}_{n}, \hat{y}_{n}\right)$ be the solution of the boundary value problem (2.6) with $g^{*}=g_{n}^{*}$ and $\alpha=1$. Similarly, let $T_{1}(\bar{g})=\left(\bar{k}, \bar{s}_{0}, \bar{\phi}, \bar{y}\right)$ be the solution of (2.6) with $g^{*}=\bar{g}^{*}$ and $\alpha=1$. From (2.7), $0 \leq$ $d \hat{\phi}_{n} / d t \leq s_{0}(A+B+k) \leq 4 m_{2} /\left(m_{1}^{*}\right)^{2},\left\|d \hat{y}_{n} / d t\right\|_{C[0,1]} \leq m_{2}$ and $\left\|d^{2} \hat{y}_{n} / d t^{2}\right\|_{C[0,1]} \leq$ $4 m_{2}^{2} /\left(m_{1}^{*}\right)^{2}$. Therefore, $\left\{\left(k_{n},\left(s_{0}\right)_{n}, \hat{\phi}_{n}, \hat{y}_{n}\right)\right\}$ has a subsequence; denote the same, such that $k_{n} \rightarrow k,\left(s_{0}\right)_{n} \rightarrow s_{0}, \hat{\phi}_{n} \rightarrow \hat{\phi}$ in $C[0,1]$, and $\hat{y}_{n} \rightarrow \hat{y}$ in $C^{1}[0,1]$ as $n \rightarrow \infty$. Since $g_{n}^{*} \rightarrow \bar{g}^{*}$ in $C[0, \pi]$ (see Lemma 1.1), by writing the differential 
equations in (2.6) as integral equations, it is easy to see that the limit $\left(k, s_{0}, \hat{\phi}, \hat{y}\right)$ also satisfies (2.6) with $g^{*}=\bar{g}^{*}$ and $\alpha=1$. From Proposition 2.2, solutions of (2.6) are unique, so $k=\bar{k}, s_{0}=\overline{s_{0}}, \hat{\phi}=\bar{\phi}$ and $\hat{y}=\bar{y}$. Hence, every sequence $\left\{T_{1}\left(g_{n}\right)\right\}$ has a subsequence that converges to the same limit $T_{1}(\bar{g})$. This implies that $T_{1}$ is continuous. In the above proof, we have also shown that for every bounded sequence $\left\{g_{n}\right\}$ in $X^{+},\left\{T_{1}\left(g_{n}\right)\right\}$ contains a convergent subsequence in $Y$. Hence $T_{1}$ is compact.

Finally denote $T_{1}(g)$ by $\left(s_{0}, k, \hat{\phi}, \hat{y}\right)$; then $\hat{\phi}$ and $\hat{y}$ will satisfy (2.6). Thus hypotheses of Proposition 2.1 will be satisfied. The proof of the lemma is complete.

Remark 4. We have shown $\left(k_{n},\left(s_{0}\right)_{n}, \hat{\phi}_{n}, \hat{y}_{n}\right) \rightarrow\left(\bar{k}, \bar{s}_{0}, \bar{\phi}, \bar{y}\right)$ in $Y$ in the above lemma when $g_{n} \rightarrow \bar{g}$ in $X^{+}$. Let the right hand side of (2.6) (a) be denoted by $f\left(k, s_{0}, \hat{\phi}, \hat{y}\right)$. Since $f\left(k_{n},\left(s_{0}\right)_{n}, \hat{\phi}_{n}, \hat{y}_{n}\right) \rightarrow f\left(\bar{k}, \bar{s}_{0}, \bar{\phi}, \bar{y}\right)$ in $C[0,1]$, we have $d \hat{\phi}_{n} / d t \rightarrow$ $d \hat{\phi} / d t$ in $C[0,1]$. This improved convergence is needed in Step 3 of the proof of Lemma 4.4.

Remark 5. As promised in Remark 1 of $\S 1$, we now explain why we need to use $g^{*}$. Ideally we would like to use $|\nabla b|$ instead of $g^{*}$ in (1.1)(c). However, when we define $T_{1}(g)$, we actually have a choice as to which function space $X$ to use for $g$. If $X=C[0, \pi]$ and we don't regularize $g$ to $g^{*}$, then the solution to (1.1) (c) with $g^{*}=g$ may not be unique. This means that it is possible for $\phi(s)=\hat{\phi}$ on an interval $\left(s_{1}, s_{2}\right)$, where $\hat{\phi}$ is a root of the right hand side of (1.1) (c). Geometrically, this is equivalent to $\partial \Omega$ containing a line segment. In such a case, $s(\phi)$ is multi-valued and $T_{1}(g)$ is not well defined. One might try letting $X=C^{1}[0, \pi]$ so that (1.1) (c) has unique solution. However, in order to use Theorem 5.1, we have to obtain a uniform bound on $\left\|g_{\lambda}\right\|_{C^{1}[0, \pi]}$ for any $g_{\lambda}$ that satisfies $\lambda T\left(g_{\lambda}\right)=g_{\lambda}$ independent of $0 \leq \lambda \leq 1$. This can be achieved if we can obtain a uniform positive lower bound for $\left(\phi_{\lambda}\right)_{t}$. Differentiating (2.6) (a), we obtain $\left(\phi_{\lambda}\right)_{t t}=\cdots-g_{\lambda}^{\prime}\left(\phi_{\lambda}\right)\left(\phi_{\lambda}\right)_{t}$. Since the lower bound of $\left(\phi_{\lambda}\right)_{t}$ is in turn dependent on the uniform bound on $\left\|g_{\lambda}\right\|_{C^{1}[0, \pi]}$, we are unable to obtain such an a priori estimate.

\section{The Map $T_{2}$}

Let $g \in X^{+}$and let $T_{1}(g)=(k, \Omega)$ be defined as in the previous section. To define $T_{2}(k, \Omega)$, we first need to solve the elliptic boundary value problem (1.1) (a), (b). Note that because $\hat{\phi} \in C^{1}[0,1]$, equations (2.5) imply that $\Omega$ is a $C^{2}$ domain. Moreover $\Omega \subset[-L, L] \times \mathbf{R}$ with $L=m_{2} / 2$ because of (2.7)(a).

Lemma 4.1. Fix $m \geq 1$. Let $k>0$ and let $\Omega \subset \mathbf{R}^{2}$ be a $C^{2}$ domain centered at the origin and symmetric about the $x$-axis. Then for any $0<\alpha<1$, there exists a unique positive function $b \in C^{2, \alpha}(\Omega) \cap C^{1, \alpha}(\bar{\Omega})$ that satisfies

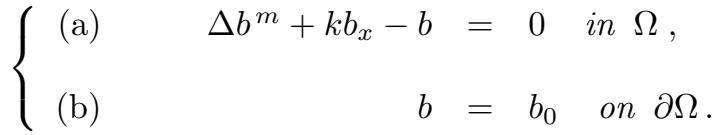

Furthermore, $b(x, y)=b(x,-y)$. Suppose $\Omega \subset[-L, L] \times \mathbf{R}$ and $0<k_{1} \leq k$. Then there exists a constant $b_{\text {min }}>0$, depending only $L$ and $k_{1}$ but not on other properties of $\Omega$, such that $b \geq b_{\min }$ in $\Omega$. 
Proof. Let $w=b^{m}$. Then (4.1) becomes

$$
\left\{\begin{aligned}
\text { (a) } \quad \Delta w+k \frac{w_{x}}{m w^{1-1 / m}}-w^{1 / m} & =0 \text { in } \Omega, \\
\text { (b) } & =b_{0}^{m} \text { on } \partial \Omega .
\end{aligned}\right.
$$

Let $\bar{w}=b_{0}^{m}$ and let $N(w)$ denote the left side of (4.2) (a). Then $N(\bar{w})<0$ in $\Omega$ and $w \leq \bar{w}$ on $\partial \Omega$. Now let $\underline{w}=\gamma e^{\delta x}$, where $\delta$ and $\gamma$ are chosen such that $k \delta>m$ and $0<\gamma e^{\delta x} \leq b_{0}^{m}$ in $\Omega$. Then

$$
N(\underline{w})=\gamma \delta^{2} e^{\delta x}+\left(\frac{k \delta}{m}-1\right) \gamma^{1 / m} e^{\delta x / m}>0 \text { in } \Omega \quad \text { and } \quad \underline{w} \leq b_{0}^{m} \text { on } \partial \Omega .
$$

Therefore, $\bar{w}$ is an upper solution and $\underline{w}$ is a lower solution of (4.2). Let $f(\xi, \vec{\eta})=$ $\xi^{1 / m}-k \eta_{1} / m \xi^{(1-1 / m)}$. Then for $\underline{w} \leq \xi \leq \bar{w}$, there exists a constant $C>0$, depending on $m, b_{0}, k, \gamma$ and $\delta$, such that $|f(\xi, \vec{\eta})| \leq C\left(1+|\vec{\eta}|^{2}\right)$. It follows from a theorem of Amann and Crandall ([1, Thm. 1]) that there exists a positive solution $w \in W^{2, p}(\Omega)$ of (4.2) for any $p>1$. By taking $p$ sufficiently large, from the Sobolev embedding theorem we have $w \in C^{1, \alpha}(\bar{\Omega})$ for any $0<\alpha<1$. Hence, $b=w^{1 / m} \in C^{1, \alpha}(\bar{\Omega})$ is a positive solution to our elliptic boundary value problem (4.1). Now we treat (4.1) (a) as a linear elliptic equation $\nabla \cdot\left(m b^{m-1} \nabla b\right)+k b_{x}-b=0$ with positive diffusion coefficient $m b^{m-1} \in C^{1, \alpha}(\bar{\Omega})$. Using standard Schauder estimates on a $C^{2}$ domain, we conclude that $b \in C^{2, \alpha}(\Omega) \cap C^{1, \alpha}(\bar{\Omega})$. This completes the existence proof of the first part of the lemma.

To prove uniqueness, let $b_{1}, b_{2}$ be two solutions to the elliptic boundary problem. Since $b_{1}, b_{2}$ are smooth, by considering a subdomain of $\Omega$ if necessary, we may assume without loss of generality that $b_{1} \geq b_{2}$ in $\Omega$. Subtracting one equation from another, we have

$$
\Delta\left(b_{1}^{m}-b_{2}^{m}\right)+k\left(b_{1}-b_{2}\right)_{x}-\left(b_{1}-b_{2}\right)=0 \text { in } \Omega, \quad b_{1}-b_{2}=0 \text { on } \partial \Omega .
$$

Integrating and applying the divergence theorem, we have

$$
\int_{\partial \Omega} \nabla\left(b_{1}^{m}-b_{2}^{m}\right) \cdot \mathbf{n}=\int_{\Omega}\left(b_{1}-b_{2}\right) .
$$

Since $b_{1}^{m} \geq b_{2}^{m} \geq 0$ in $\Omega$ and $b_{1}=b_{2}$ on $\partial \Omega$, the left side of the above equation is nonpositive, while the right side is positive. Hence, $b_{1}=b_{2}$ and solutions to (4.1) are unique. Since $\Omega$ is symmetric about the $x$-axis, $b(x,-y)$ is also a solution of (4.1). From uniqueness, $b(x, y)=b(x,-y)$.

To prove the second half of the lemma, we simply choose $\delta$ and $\gamma$ in the definition of $\underline{w}$ above such that $k_{1} \delta>m$ and $0<\gamma e^{\delta L} \leq b_{0}^{m}$, and we let $b_{\min }=\min _{\Omega} \underline{w}^{1 / m}$. Then $b_{\text {min }}$ depends only on $m, L, k_{1}$ and $b_{0}$ and $b \geq b_{\min }$ in $\Omega$. The proof of the lemma is complete.

Let $b(x, y)$ be the solution obtained in Lemma 4.1. Recalling that $t=s / s_{0}$, where $s$ is the arc length, we can regard $|\nabla b|$ as a function of $t \in[0,1]$ in the upper half of $\partial \Omega$. Let $g \in X^{+}$; then from Proposition 2.2, $d \hat{\phi} / d t>0$, so that the inverse function $t(\hat{\phi})$ can be defined and is continuous. Let $T_{2}(k, \Omega)=|\nabla b|(t(\phi))$, which clearly belongs to $X^{+}$. We need to show that $T_{2}$ is continuous in the sense that if $T_{1}\left(g_{n}\right)=\left(k_{n}, \Omega_{n}\right) \rightarrow(\bar{k}, \bar{\Omega})$ in $Y$, then $T_{2}\left(k_{n}, \Omega_{n}\right) \rightarrow T_{2}(\bar{k}, \bar{\Omega})$ in $X$ as $n \rightarrow \infty$. ( $Y$ is defined in Lemma 3.1). Together with Lemma 3.1. $T=T_{2} \circ T_{1}: X^{+} \rightarrow X^{+}$is continuous and compact. A crucial part of the proof is to control $\left\|b_{n}\right\|_{C^{1, \alpha}(\bar{\Omega})}$, where 
$b_{n}$ is the solution of (4.1) with $k=k_{n}$ and $\Omega=\Omega_{n}$. This is done in Lemma 4.3. and the proof of continuity of $T_{2}$ is given in Lemma 4.4. To prove Lemma 4.3, we need the following lemma.

Lemma 4.2. Let $\Omega$ be a $C^{2}$ convex domain which is symmetric about the $x$-axis. Let its boundary curvature $\kappa$ satisfy $0<\kappa \leq \kappa_{0}$. Then there exists a disk $U$ with radius $1 / 2 \kappa_{0}$ such that for any point $P \in \partial \Omega, U$ may be placed inside $\Omega$ and touches $\partial \Omega$ only at $P$.

Proof. Without loss of generality, we may assume that $P=\left(x_{1}, 0\right)$ is the furthermost right point on $\Omega$ lying on the $x$-axis. Let $U$ be a circle of radius $1 / 2 \kappa_{0}$. Since $\partial U$ has a constant curvature of $2 \kappa_{0}$, it is clear that in a small neighborhood of $P, U$ touches $\partial \Omega$ only at $P$. Now suppose $\partial \Omega$ comes back and intersects $U$ from outside for the first time at the point $Q=\left(x_{2}, y_{2}\right)$. Because $\Omega$ is symmetric about the $x$-axis, we may assume that $y_{2}>0$. Recall that $\phi$ represents the normal angle of a domain. Let $\left.\phi\right|_{Q}=\phi_{1}$ for $U$ and $\left.\phi\right|_{Q}=\phi_{2}$ for $\Omega$. Then $0<\phi_{1} \leq \phi_{2} \leq \pi$. Let $(x(s), y(s))$ represent the location of a boundary point in rectangular coordinates where $s$ is the arc length measured counterclockwise from $P$. The equations

$$
\left\{\begin{aligned}
\text { (a) } & \frac{d x}{d s}=-\sin \phi \\
\text { (b) } & \frac{d y}{d s}=\cos \phi
\end{aligned}\right.
$$

hold for both $\partial U$ and $\partial \Omega$. Moreover, their respective normal angles satisfy $0 \leq \phi \leq$ $\pi$ from $P$ to $Q$. Integrating (4.3) (a) along the boundary of $U$ from $P$ to $Q$ counterclockwise, we have $-\int_{x_{1}}^{x_{2}} d x=\int_{0}^{\phi_{1}} \sin \phi(d s / d \phi) d \phi=\int_{0}^{\phi_{1}} \sin \phi / 2 \kappa_{0} d \phi$. Likewise, integrating (4.3) (a) along $\partial \Omega$ from $P$ to $Q$, we have $-\int_{x_{1}}^{x_{2}} d x=\int_{0}^{\phi_{2}} \sin \phi(d s / d \phi) d \phi$ $=\int_{0}^{\phi_{2}} \sin \phi / \kappa d \phi$. Therefore,

$$
\int_{0}^{\phi_{1}} \frac{\sin \phi}{2 \kappa_{0}} d \phi=\int_{0}^{\phi_{2}} \frac{\sin \phi}{\kappa} d \phi \geq \int_{0}^{\phi_{1}} \frac{\sin \phi}{\kappa} d \phi \geq \int_{0}^{\phi_{1}} \frac{\sin \phi}{\kappa_{0}} d \phi
$$

which is a contradiction. Therefore, $\partial \Omega$ cannot intersect $U$ at $Q$. The proof of the lemma is complete.

Lemma 4.3. Let $\Omega \subset[-L, L] \times \mathbf{R}$ satisfy the hypotheses of Lemma 4.2 and let $0<k_{1} \leq k \leq k_{2}$. Then there exists $0<\alpha<1$ and $M_{1, \alpha}>0$, which depends on $L, k_{1}, k_{2}$ and $\kappa_{0}$ only but not on other properties of $\Omega$, such that the unique positive solution $b$ from Lemma 4.1 satisfies $\|b\|_{C^{1, \alpha}(\bar{\Omega})} \leq M_{1, \alpha}$.

Proof. We regard (1.1) (a) as a linear elliptic equation $\nabla \cdot\left(m b^{m-1} \nabla b\right)+k b_{x}-b=0$ with diffusion coefficient $m b^{m-1}$. From Lemma 4.1 there exists a constant $b_{\text {min }}>$ 0 , depending only on $L$ and $k_{1}$, such that $b_{\text {min }} \leq b \leq b_{0}$. It is clear that a $C^{2}$ convex domain will satisfy the uniform exterior cone condition (see [10, p. 205]) since a cone $V$ with vertex angle $\pi / 4$ can be attached to any point on $\partial \Omega$ and lie outside of $\Omega$. At any point $x_{0} \in \partial \Omega$, let $B\left(x_{0}, R_{0}\right)$ be a disk with radius $R_{0}=1 / 2 \kappa_{0}$ constructed according to Lemma 4.2. We now apply Theorem 8.27 in [10] to (1.1). Since there is no source term and $b=b_{0}$ on $\partial \Omega$, we have, using the notation in Theorem 8.27 of [10], $k=0, \sigma=0, \lambda=m b_{m i n}^{m-1}, \Lambda=m b_{0}^{m-1}$ and $\nu=\sqrt{k_{2}^{2}+1} / \lambda$. Hence, $b$ is Hölder continuous along a strip $S$ of uniform width covering $\partial \Omega$ with Hölder exponent $\alpha$ and Hölder norm of $b$ in $S$ depending only on $\lambda, \Lambda, \nu, R_{0}$ and 
the cone $V$. Since $b_{\text {min }}$ depends only on $k_{1}, L$, and $\alpha, S$ depend only on $L, k_{1}$, $k_{2}$ and $\kappa_{0}$, together with a similar Hölder estimate in the interior of $\Omega$ (see [10, Thm. 8.22]), $b \in C^{\alpha}(\bar{\Omega})$, where $\alpha$ and $\|b\|_{C^{\alpha}(\bar{\Omega})}$ depend only on $L, k_{1}, k_{2}$ and $\kappa_{0}$. Once we have a uniform Hölder norm bound on $b$, Theorem 8.33 of [10] allows us to bootstrap to the $C^{1, \alpha}$ norm bound for $b$. In view of the last line on page 210 of [10], such a higher norm bound depends only on the constants $L, k_{1}, k_{2}$ and $\kappa_{0}$. The proof of the lemma is complete.

Lemma 4.4. Let $g_{n} \rightarrow \bar{g}$ in $X^{+}$as $n \rightarrow \infty$. Let $T_{1}\left(g_{n}\right)=\left(k_{n}, \Omega_{n}\right)$ and $T_{1}(\bar{g})=$ $\left(\bar{k}, \bar{\Omega}_{n}\right)$. Then $T_{2}\left(k_{n}, \Omega_{n}\right) \rightarrow T_{2}(\bar{k}, \bar{\Omega})$ in $X$ as $n \rightarrow \infty$.

Proof. The proof of this lemma is rather technical and is given in Appendix B.

\section{Proof of Theorem 1.1}

Theorem 5.1 (Schaefer's Theorem). Let $Z^{+}$be a cone of a Banach space $Z$ and let $\mathcal{A}: Z^{+} \rightarrow Z^{+}$be a continuous and compact mapping. Assume that the set $\left\{u \in Z^{+} \mid u=\lambda \mathcal{A}[u]\right.$ for some $\left.0 \leq \lambda \leq 1\right\}$ is bounded. Then $\mathcal{A}$ has a fixed point in $Z^{+}$.

Proof. See [8, Chaper 9.2.2], which is stated for a Banach space. The proof there also works when $Z$ is replaced by $Z^{+}$.

Lemma 5.1. Let $\lambda T\left(g_{\lambda}\right)=g_{\lambda}$, where $0<\lambda \leq 1$. Then $s_{0}(\lambda) \geq m_{1}(\lambda)$, where $m_{1}(\lambda)$ is the unique positive root of the equation

$$
q_{\lambda}(x)=-\frac{x^{2}}{m b_{0}^{m-2} \pi}+\frac{1}{\lambda}\left(\frac{2}{x}-V_{d} x-2 \pi\right)=0 .
$$

Proof. Let $T_{1}(g)=\left(s_{0}, k, \phi, y\right)=(k, \Omega)$ and let $T(g)=T_{2}(k, \Omega)=|\nabla b|(\phi)$. For the time being, we do not assume that $g$ is a fixed point of $\lambda T$. We first integrate equation (4.1) (a) over $\Omega$. Since $b \leq b_{0}$ and $\mathbf{n}=\nabla b /|\nabla b|$, the isoparametric inequality and divergence theorem imply that

$$
\begin{aligned}
b_{0} \frac{\left(2 s_{0}\right)^{2}}{4 \pi} & \geq b_{0}|\Omega| \geq \int_{\Omega} b=m b_{0}^{m-1} \int_{\partial \Omega} \frac{\partial b}{\partial n}=m b_{0}^{m-1} \int_{\partial \Omega}|\nabla b| d s \\
& =2 m b_{0}^{m-1} s_{0} \int_{0}^{1}|\nabla b|(t) d t=2 m b_{0}^{m-1} \int_{0}^{\pi} \frac{|\nabla b|(\phi)}{\kappa(\phi)} d \phi,
\end{aligned}
$$

where $\kappa=d \phi / d s$. Since $y(0)=y(1)=0$, integrating equation (2.6) (a) with $\alpha=1$, we have

$$
\pi=s_{0} A-s_{0} \int_{0}^{1} g^{*}(\phi(t)) d t=s_{0} A-\int_{0}^{s_{0}} g^{*}(\phi(s)) d s=s_{0} A-\int_{0}^{\pi} \frac{g^{*}(\phi)}{\kappa(\phi)} d \phi .
$$

Now suppose $\lambda T\left(g_{\lambda}\right)=g_{\lambda}$ and $T\left(g_{\lambda}\right)=\left|\nabla b_{\lambda}(\phi)\right|$, where we have used $b_{\lambda}$ to emphasize the dependence of $b$ on $\lambda$. From our hypothesis, $g_{\lambda}=\lambda\left|\nabla b_{\lambda}\right|(\phi)$. From Remark 1 in $\S 1, g_{\lambda}^{*}(\phi)=\lambda\left[\left|\nabla b_{\lambda}\right|(\phi)\right]^{*} \leq \lambda\left|\nabla b_{\lambda}\right|(\phi)$. Therefore, (5.3) and the definition of $A$ imply that

$$
\lambda \int_{0}^{\pi} \frac{\left|\nabla b_{\lambda}\right|(\phi)}{\kappa_{\lambda}(\phi)} d \phi \geq \frac{1}{s_{0}(\lambda)}-\frac{V_{d}}{2} s_{0}(\lambda)-\pi .
$$

Combining this with (5.2), we have $q_{\lambda}\left(s_{0}\right) \leq 0$. It can be proved (see proof of Lemma 2.1) that $q_{\lambda}(x)$ decreases from $\infty$ to $-\infty$ on the interval $(0, \infty)$. Therefore, $m_{1}(\lambda) \leq s_{0}(\lambda)$. The proof of the lemma is complete. 
Lemma 5.2. Let $\lambda T\left(g_{\lambda}\right)=g_{\lambda}$, where $0<\lambda \leq 1$. Then there exist $\alpha \in(0,1)$ and $C$, which depend only on $m, b_{0}$ and $V_{d}$ but not on $\lambda$, such that $\left\|g_{\lambda}\right\|_{X} \leq C$.

Proof. Differentiating (5.1) with respect to $\lambda$ and rearranging, we have

$$
\frac{d m_{1}(\lambda)}{d \lambda}=\frac{-\frac{m_{1}^{2}(\lambda)}{\lambda m b_{0}^{m-2} \pi}}{\frac{2 m_{1}(\lambda)}{m b_{0}^{m-2} \pi}+\frac{1}{\lambda}\left(\frac{2}{m_{1}^{2}(\lambda)}+V_{d}\right)}<0 .
$$

Therefore, $m_{1} \leq m_{1}(\lambda) \leq s_{0}(\lambda)$ for $0<\lambda \leq 1$, where $m_{1}$ is defined in Lemma 2.1. It can also be checked that $s_{0}(\lambda) \leq m_{2}$ (see the proof of Lemma 2.1). Since $\kappa_{\lambda}(\phi)=s_{0}(\lambda)\left[A_{\lambda}+\left(B_{\lambda}-k_{\lambda}\right) \cos \phi-g_{\lambda}^{*}(\phi)\right] \geq 0$, we have $A_{\lambda}+\left(B_{\lambda}-k_{\lambda}\right) \cos \phi \geq$ $g_{\lambda}^{*}(\phi) \geq 0$. Let $\phi=0$; then $k_{\lambda} \leq A_{\lambda}+B_{\lambda}=2 / s_{0}^{2}(\lambda) \leq 2 / m_{1}^{2}$. Also, $d \hat{\phi}_{\lambda} / d t \leq$ $s_{0}(\lambda)\left(A_{\lambda}+B_{\lambda}+k_{\lambda}\right) \leq 4 m_{2} / m_{1}^{2}$. Therefore, the bounds in (2.7) hold for $s_{0}(\lambda), k_{\lambda}$ and $\hat{\phi}_{\lambda}$, with $m_{1}^{*}$ replaced by $m_{1}$. These bounds are independent of $0<\lambda \leq 1$. Let $\kappa_{0}=4 m_{2} / m_{1}^{2}$ in Lemma 4.3. Then Lemma 4.3 implies that $\left\|b_{\lambda}\right\|_{C^{1, \alpha}\left(\overline{\Omega_{\lambda}}\right)} \leq M_{1, \alpha}$ with $\alpha$ and $M_{1, \alpha}$ again independent of $\lambda \in(0,1]$. Since $\left\|g_{\lambda}\right\|_{X}=\left\|\lambda \nabla b_{\lambda}\right\|_{X}$, we can let $C=M_{1, \alpha}$. The proof of the lemma is complete.

From Lemmas 3.1 and 4.4 $T: X^{+} \rightarrow X^{+}$is continuous and compact. From Lemma 5.2, the hypotheses of Theorem 5.1 are satisfied. Therefore, $T$ has a fixed point which solves (1.1) and is our traveling domain solution. From (1.3), $g^{*}(\phi)=$ $[|\nabla b|]^{*}(\phi)$ is $C^{\infty}$. Equation (2.6) (a) with $\alpha=1$ implies that $\hat{\phi} \in C^{\infty}[0,1]$ and $\hat{y} \in C^{\infty}[0,1]$ for our fixed point. From Proposition 2.1. the domain $\Omega$ is $C^{\infty}$ and equation (4.1) implies that $b \in C^{\infty}(\bar{\Omega})$. The proof of Theorem 1.1 is complete.

\section{Appendix A: Proof of Proposition 2.2}

Lemma 6.1. Let $\alpha=0$; then there exists a solution to system (2.6) with

$$
\begin{aligned}
\text { (a) } s_{0}=\frac{-\pi+\sqrt{\pi^{2}+2 V_{d}}}{V_{d}}, & \text { (b) } k=V_{d}+\frac{\pi}{s_{0}}, \\
\text { (c) } \hat{\phi}(t)=\pi t, \quad \text { and } & \text { (d) } \hat{y}(t)=\frac{s_{0}}{\pi} \sin (\pi t) .
\end{aligned}
$$

Proof. This solution corresponds to the domain $\Omega$ being a circle with radius $s_{0} / \pi$ and can be verified by direct substitution. Note that $A=\pi / s_{0}$ and $k=B$ in this case.

Lemma 6.2. The solution to (2.6) is unique when $\alpha=0$.

Proof. Let $\left(s_{0}, k, \hat{\phi}, \hat{y}\right)$ be a solution to (2.6) ; then $\kappa(\hat{\phi}(t)) \neq 0$ where $\kappa(\phi)$ is the right side of equation (2.6) (a). For if $\kappa\left(\hat{\phi}\left(t_{1}\right)\right)=0$, then $\hat{\phi}_{1}(t)=\hat{\phi}\left(t_{1}\right)$ is a constant solution of (2.6) (a) that equals $\hat{\phi}(t)$ at $t=t_{1}$. By the uniqueness of the solution to initial value problems for ODE, $\hat{\phi}(t)=\hat{\phi}\left(t_{1}\right)$. But then the boundary conditions $\hat{\phi}(0)=0$ and $\hat{\phi}(1)=\pi$ will not be satisfied simultaneously. Therefore, $d \hat{\phi} / d t>0$ and $0 \leq \hat{\phi}(t) \leq \pi$ on $[0,1]$.

From (2.6), we have

$$
\frac{d \hat{\phi}}{d t}=s_{0} A+(B-k) \frac{d \hat{y}}{d t} .
$$

Integrating this equation from 0 to 1 , we have $A s_{0}=\pi$. Since $A=1 / s_{0}^{2}-V_{d} / 2, s_{0}$ is given by (6.1) (a), so all solutions of (2.6) have the same $s_{0}$. 
We now show that $k$ is also the same for all solutions of (2.6). Integrating the above displayed equation, we have $\hat{\phi}(t)=s_{0} A t+(B-k) \hat{y}(t)$. From (2.6) (b), we have $d \hat{y} / d t=s_{0} \cos \hat{\phi}=s_{0} \cos \left(s_{0} A t+(B-k) \hat{y}\right)$. This motivates us to consider the initial value problem

$$
\frac{d z}{d t}=s_{0} \cos \left(s_{0} A t+(B-u) z\right), \quad z(0, u)=0,
$$

where $u>0$ is a parameter. Differentiating with respect to $u$, we have

$$
\frac{d z_{u}}{d t}+s_{0}(B-u)(\sin \psi) z_{u}=s_{0}(\sin \psi) z \quad \text { and } \quad z_{u}(0, u)=0,
$$

where $\psi=s_{0} A t+(B-u) z$. We want to consider the function $z(1, u)$ for $u>0$. At $u=k, z(t, k)=\hat{y}(t)$ so that $\psi=\hat{\phi}$, which lies between 0 and $\pi$. Hence, $\sin (\psi) \geq 0$. Also, $z(t, k)=\hat{y}(t) \geq 0$. Consequently, $s_{0}(\sin \psi) z \geq 0$ and equation (6.2) becomes an inequality of the form $d z_{u} / d t+f(t) z_{u} \geq 0, z_{u}(0, k)=0$. Since the inequality is strict for some $t$, by integrating this over $[0,1]$ we have $z_{u}(1, k)>0$. The above analysis implies that whenever $u=k$ corresponds to a solution of (2.6), then $z(1, k)=0$ and $z_{u}(1, k)>0$. Thus, the graph $z(1, u)$ versus $u$ can cross zero at most once, which implies that $k$ is the same for any solution of (2.6). With $s_{0}$ and $k$ now being unique, the uniqueness of $\hat{y}$ and $\hat{\phi}$ are immediate. The proof of the lemma is complete.

Lemma 6.3. Suppose $g^{*} \in C^{1}[0, \pi]$ is a nonnegative function and let $\left\|g^{*}\right\|_{C[0, \pi]} \leq$ $M_{1}$. Recall the definitions of $m_{1}$ and $m_{2}$ from Lemma 2.1. Then for $0 \leq \alpha \leq 1$, (2.6) has a solution $s_{0}, k, \hat{\phi} \in C^{2}[0,1]$, and $\hat{y} \in C^{3}[0,1]$, where

$$
m_{1}^{*} \leq s_{0} \leq m_{2}, \quad V_{d} \leq k \leq \frac{2}{m_{1}^{* 2}}, \quad 0<\kappa=\frac{d \hat{\phi}}{d t} \leq \frac{4 m_{2}}{m_{1}^{* 2}},
$$

and $m_{1}^{*}=\left(-\pi+\sqrt{\pi^{2}+2 V_{d}+4 M_{1}}\right) /\left(V_{d}+2 M_{1}\right)$.

Proof. Let $\Phi=\Phi\left(t, k, s_{0}\right)$ and $Y=Y\left(t, k, s_{0}, \alpha\right)$ be the solutions to equations (2.6) (a) and (2.6) (b), respectively, with initial conditions $\Phi(0)=0$ and $Y(0)=0$. For a given $\alpha \in(0,1]$, finding a solution to (2.6) is equivalent to solving the algebraic equations

$$
\left\{\begin{aligned}
F\left(k, s_{0}, \alpha\right) & \equiv \Phi\left(1, k, s_{0}, \alpha\right)-\pi=0, \\
G\left(k, s_{0}, \alpha\right) & \equiv Y\left(1, k, s_{0}, \alpha\right)=0
\end{aligned}\right.
$$

for $s_{0}$ and $k$. We know there is an explicit unique solution when $\alpha=0$, and we like to compute the degree of the map $(F, G)$ at this solution. From (2.6) (a), we have

$$
\frac{d}{d t}\left(\frac{\partial \Phi}{\partial k}\right)=-s_{0} \cos \Phi-(B-k) s_{0} \sin \Phi \frac{\partial \Phi}{\partial k}-s_{0} \alpha g^{* \prime}(\Phi) \frac{\partial \Phi}{\partial k}
$$

with $\partial \Phi(0) / \partial k=0$. When $\alpha=0$, from Lemmas 6.1 and 6.2 we have $B=k$ and $\Phi=\pi t$. Hence, 6.5.5 becomes

$$
\frac{d}{d t}\left(\frac{\partial \Phi}{\partial k}\right)=-s_{0} \cos (\pi t) .
$$


Integrating, we obtain $\partial \Phi / \partial k=-s_{0} \sin (\pi t) / \pi$, and hence $\partial F / \partial k=0$ when $\alpha=0$. Similarly,

$$
\begin{aligned}
\frac{d}{d t}\left(\frac{\partial \Phi}{\partial s_{0}}\right)= & A+(B-k) \cos \Phi-\alpha g^{*}(\Phi) \\
& +s_{0}\left[\frac{\partial A}{\partial s_{0}}+\frac{\partial B}{\partial s_{0}} \cos \Phi-(B-k) \sin \Phi \frac{\partial \Phi}{\partial s_{0}}-\alpha g^{* \prime}(\Phi) \frac{\partial \Phi}{\partial s_{0}}\right] .
\end{aligned}
$$

When $\alpha=0$, since $A=1 / s_{0}^{2}-V_{d} / 2$ and $B=A+V_{d}$, the above equation may be simplified to

$$
\begin{aligned}
\frac{d}{d t}\left(\frac{\partial \Phi}{\partial s_{0}}\right) & =\left(\frac{1}{s_{0}^{2}}-\frac{V_{d}}{2}\right)+s_{0}\left[-\frac{2}{s_{0}^{3}}-\frac{2}{s_{0}^{3}} \cos \Phi\right] \\
& =-\frac{1}{s_{0}^{2}}-\frac{V_{d}}{2}-\frac{2}{s_{0}^{2}} \cos (\pi t)
\end{aligned}
$$

with $\partial \Phi(0) / \partial s_{0}=0$. Solving this initial value problem, we have $\partial \Phi(1) / \partial s_{0}=$ $\partial F / \partial s_{0}=-1 / s_{0}^{2}-V_{d} / 2$. To continue, we differentiate (2.6) (b) with respect to $k$ to obtain

$$
\frac{d}{d t}\left(\frac{\partial Y}{\partial k}\right)=-s_{0} \sin \Phi \frac{\partial \Phi}{\partial k}
$$

with $\partial Y(0) / \partial k=0$. From above, we know that when $\alpha=0, \partial \Phi / \partial k=-s_{0} \sin (\pi t) / \pi$. Therefore,

$$
\frac{d}{d t}\left(\frac{\partial Y}{\partial k}\right)=\frac{s_{0}^{2}}{\pi} \sin ^{2}(\pi t)
$$

with $\partial Y(0) / \partial k=0$. Integrating, we obtain $\partial Y(1) / \partial k=\partial G / \partial k=s_{0}^{2} /(2 \pi)$ when $\alpha=0$. Putting everything together, at the unique solution of (6.4) when $\alpha=0$, we have

$$
\operatorname{det}\left(\begin{array}{cc}
F_{k} & F_{s_{0}} \\
G_{k} & G_{s_{0}}
\end{array}\right)=\operatorname{det}\left(\begin{array}{cc}
0 & -1 / s_{0}^{2}-V_{d} / 2 \\
s_{0}^{2} / 2 \pi & G_{s_{0}}
\end{array}\right)=\frac{1}{4 \pi}\left(2+V_{d} s_{0}^{2}\right)>0 .
$$

Let $\mathcal{F}=(F, G)$ and let $B_{R}$ be the open ball centered at the origin with radius $\sqrt{k^{2}+s_{0}^{2}}=R$. If $R$ is large, it will contain the unique solution of (6.4) for $\alpha=0$. Then equation (6.7) allows us to conclude that when $\alpha=0$, we have $\operatorname{deg}\left(\mathcal{F}, B_{R}, 0\right)=$ 1 for any sufficiently large $R$.

In order to prove that (6.4) has a solution for $0 \leq \alpha \leq 1$, we need to establish a priori bounds on $s_{0}$ and $k$ for $\alpha \in[0,1]$. To begin, we have $d \hat{\phi} / d t>0$ (see the beginning of the proof of Lemma [.2. $), 0 \leq \hat{\phi} \leq \pi, 0 \leq g^{*}(\hat{\phi}) \leq M_{1}$, and $\hat{y} \geq 0$ on $[0,1]$. Integrating (2.6) (a) with respect to $t$, we have

$$
\hat{\phi}=s_{0} A t+(B-k) \hat{y}-\alpha s_{0} \int_{0}^{t} g^{*}(\hat{\phi}(\xi)) d \xi .
$$

Evaluating at $t=1$, we have

$$
\left[\frac{V_{d}}{2}+\alpha \int_{0}^{1} g^{*}(\hat{\phi}(\xi)) d \xi\right] s_{0}^{2}+\pi s_{0}-1=0 .
$$

Hence,

$$
s_{0}=\frac{-\pi+\sqrt{\pi^{2}+2 V_{d}+4 \alpha \int_{0}^{1} g^{*}(\hat{\phi}(\xi)) d \xi}}{V_{d}+2 \alpha \int_{0}^{1} g^{*}(\hat{\phi}(\xi)) d \xi} \leq \frac{-\pi+\sqrt{\pi^{2}+2 V_{d}}}{V_{d}}=m_{2} .
$$


The last inequality follows because the equation $a x^{2}+b x-c=0$, where $a, b$ and $c$ are positive, has a unique positive root $x=x(a)$. Since $\partial x / \partial a=-x^{2} /(2 a x+b)<0, x$ is decreasing in $a$. The right side of the inequality in (6.9) is the positive root of the equation $\left(V_{d} / 2\right) s_{0}^{2}+\pi s_{0}-1=0$, and therefore (6.9) follows. Let $M_{1}=\left\|g^{*}\right\|_{C[0, \pi]}$; we have $\alpha \int_{0}^{1} g^{*}(\hat{\phi}(\xi)) d \xi \leq M_{1}$. From (6.9),$\left.s_{0} \geq \sqrt{\pi^{2}+2 V_{d}+4 M_{1}}\right) /\left(V_{d}+2 M_{1}\right)=$ $m_{1}^{*}$. Hence, $m_{1}^{*} \leq s_{0} \leq m_{2}$.

We now turn to estimate $k$. Evaluating (2.6) (a) at $t=0$, we have

$$
s_{0}\left[A+B-k-\alpha g^{*}(0)\right]=d \hat{\phi}(0) / d t \geq 0 .
$$

Hence, $k \leq A+B=2 / s_{0}^{2} \leq 2 / m_{1}^{* 2}$. Evaluating (2.6) (a) at $t=1$, we have $s_{0}\left[A-B+k-\alpha g^{*}(\pi)\right]=d \hat{\phi}(1) / d t \geq 0$. Thus, $k \geq V_{d}+\alpha g^{*}(\pi) \geq V_{d}$.

We now define the domain

$$
\mathcal{U}=\left\{\left(k, s_{0}\right) \mid \frac{V_{d}}{2}<k<\frac{4}{m_{1}^{* 2}}, \frac{m_{1}^{*}}{2}<s_{0}<2 m_{2}\right\} .
$$

From the above a priori bounds, $\operatorname{deg}(\mathcal{F}, \mathcal{U}, 0)=\operatorname{deg}\left(\mathcal{F}, B_{R}, 0\right)=1$ by the excision property of degree and $\mathcal{F} \neq 0$ on $\partial \mathcal{U}$ for $0 \leq \alpha \leq 1$. Hence, $\operatorname{deg}(\mathcal{F}, \mathcal{U}, 0)=1$ for any $\alpha \in[0,1]$. In other words a solution exists for (6.4), which is equivalent to (2.6). From (2.6) (a), $\kappa=d \hat{\phi} / d t \leq s_{0}[A+B+k] \leq 4 m_{2} / m_{1}^{* 2}$. It is also clear that $\hat{\phi} \in C^{2}[0,1]$ and $\hat{y} \in C^{3}[0,1]$. The proof of the lemma is complete.

Lemma 6.4. Let the hypotheses of Lemma 6.3 be satisfied. Then the solution to (2.6), which is shown to exist in Lemma 6.3, is unique.

Proof. Let $\kappa=A+(B-k) \cos \phi-\alpha g^{*}(\phi)$. Then because $g^{*}$ is smooth, the solution to (2.6) (a) is unique and $\kappa>0$ on $[0, \pi]$. Integrating (2.6), we have

$$
\begin{cases}\text { (a) } & s_{0}-\int_{0}^{\pi} \frac{1}{\kappa} d \phi=0, \\ \text { (b) } & \int_{0}^{\pi} \frac{\cos \phi}{\kappa} d \phi=0 .\end{cases}
$$

We shall show that (6.10) has unique solutions. Let $z=1 / s_{0}^{2}$ and rewrite (6.10) as

$$
\left\{\begin{aligned}
\frac{1}{\sqrt{z}}-\int_{0}^{\pi} \frac{1+\cos \phi}{\kappa} d \phi & =0, \\
\int_{0}^{\pi} \frac{\cos \phi}{\kappa} d \phi & =0 .
\end{aligned}\right.
$$

Also, $A=z-V_{d} / 2$ and $B=z+V_{d} / 2$.

Let

$$
I(z, k)=2 \sqrt{z}-\int_{0}^{\pi} \log \left[A+(B-k) \cos \phi-\alpha g^{*}(\phi)\right] d \phi .
$$

Direct calculations yield

$$
\left\{\begin{array}{l}
\frac{\partial I}{\partial z}=\frac{1}{\sqrt{z}}-\int_{0}^{\pi} \frac{1+\cos \phi}{\kappa} d \phi \\
\frac{\partial I}{\partial k}=\int_{0}^{\pi} \frac{\cos \phi}{\kappa} d \phi .
\end{array}\right.
$$


Finding a critical point of $I$ is equivalent to solving 6.11). Upon further calculations, we have

$$
\left\{\begin{aligned}
\frac{\partial^{2} I}{\partial z^{2}} & =-\frac{1}{2 z^{3 / 2}}+\int_{0}^{\pi} \frac{(1+\cos \phi)^{2}}{\kappa^{2}} d \phi \\
\frac{\partial^{2} I}{\partial k^{2}} & =\int_{0}^{\pi} \frac{\cos ^{2} \phi}{\kappa^{2}} d \phi \\
\frac{\partial^{2} I}{\partial z \partial k} & =-\int_{0}^{\pi} \frac{\cos \phi(1+\cos \phi)}{\kappa^{2}} d \phi
\end{aligned}\right.
$$

Define the Hessian matrix

$$
H=\left(\begin{array}{cc}
I_{z z} & I_{z k} \\
I_{z k} & I_{k k}
\end{array}\right)
$$

After some algebraic manipulations, we find that

$$
\operatorname{det}(H)=\int_{0}^{\pi} \frac{\cos ^{2} \phi}{\kappa^{2}} d \phi\left(\int_{0}^{\pi} \frac{1}{\kappa^{2}} d \phi-\frac{s_{0}^{3}}{2}\right)-\left(\int_{0}^{\pi} \frac{\cos \phi}{\kappa^{2}} d \phi\right)^{2} .
$$

Let $\beta=s_{0} / \pi$. From $(=6.10$ (b),

$$
\int_{0}^{\pi} \frac{\cos \phi}{\kappa^{2}} d \phi=\int_{0}^{\pi} \frac{\cos \phi}{\kappa}\left(\frac{1}{\kappa}-\beta\right) d \phi .
$$

From Cauchy-Schwartz's inequality, (6.10)(a) and the definition of $\beta$, we have

$$
\begin{aligned}
\left(\int_{0}^{\pi} \frac{\cos \phi}{\kappa^{2}} d \phi\right)^{2} & \leq \int_{0}^{\pi} \frac{\cos ^{2} \phi}{\kappa^{2}} d \phi \int_{0}^{\pi}\left(\frac{1}{\kappa}-\beta\right)^{2} d \phi \\
& =\int_{0}^{\pi} \frac{\cos ^{2} \phi}{\kappa^{2}} d \phi \quad\left(\int_{0}^{\pi} \frac{1}{\kappa^{2}} d \phi-\frac{s_{0}^{2}}{\pi}\right) .
\end{aligned}
$$

Since $s_{0} \leq m_{2}=\left(-\pi+\sqrt{\pi^{2}+2 V_{d}}\right) / V_{d}<2 / \pi$, we have $s_{0}^{2} / \pi>s_{0}^{3} / 2$, which implies that $\operatorname{det}(H)>0$ at any critical point of $I$.

Let $\hat{\mathcal{F}}=\left(I_{z}, I_{k}\right)$ and consider it as a function of $(z, k, \alpha)$. From above, at any critical point $\left(z_{0}, k_{0}\right)$ of $I$, we have $\hat{\mathcal{F}}\left(z_{0}, k_{0}, \alpha\right)=0$ and $\operatorname{det}\left(\hat{\mathcal{F}}^{\prime}\right)\left(z_{0}, k_{0}, \alpha\right)=$ $\operatorname{det}(H)\left(z_{0}, k_{0}, \alpha\right)>0$, where ' means derivatives with respect to $z$ and $k$. For any $\alpha \in[0,1]$, a solution $\left(z_{0}, k_{0}\right)$ to $\hat{F}(z, k, \alpha)=0$ exists by assumption. Since $\operatorname{det}\left(\hat{F}^{\prime}\right)>0$ at any such solution, the implicit function theorem implies that $\left(z_{0}, k_{0}\right)$ is a $C^{1}$ function of $\alpha$ and never bifurcates. Since we also have a priori bounds on $z_{0}$ and $k_{0}$ for $\alpha \in[0,1]$, the curve $\left(z_{0}(\alpha), k_{0}(\alpha)\right)$ exists for $\alpha \in[0,1]$. If there are 2 solutions at some $\alpha=\alpha_{0}$ where $\alpha_{0} \in[0,1]$, we will have 2 distinct solution curves going back to $\alpha=0$, since the curves do not bifurcate. Hence, there are 2 distinct solutions for $\alpha=0$ which contradict Lemma 6.2. Therefore there is a unique $C^{1}$ solution curve $\left(z_{0}(\alpha), k_{0}(\alpha)\right)$ for $\alpha \in[0,1]$. With $s_{0}$ and $k$ being unique, since $g^{*} \in C^{1}[0, \pi]$, solutions of $(2.6)$ are clearly unique. The proof of the lemma is complete. 


\section{Appendix B: Proof of Lemma 4.4}

Let $T_{1}\left(g_{n}\right)=\left(k_{n}, \Omega_{n}\right) \rightarrow T_{1}(\bar{g})=\left(\bar{k}, \Omega_{0}\right)$ as $n \rightarrow \infty$. To show that $T_{2}\left(k_{n}, \Omega_{n}\right)$ converges to $T_{2}\left(\bar{k}, \Omega_{0}\right)$, we shall prove that every sequence of $T_{2}\left(k_{n}, \Omega_{n}\right)$ has a further subsequence that converges to $T_{2}\left(\bar{k}, \Omega_{0}\right)$ as $n \rightarrow \infty$. There are three steps to the proof.

Step 1 . There exists a subsequence $\left\{b_{n_{j}}\right\}$ such that $\left\|b_{n_{j}}-\bar{b}\right\|_{C^{1}(K)} \rightarrow 0$ as $j \rightarrow 0$ on every compact subset $K$ in the interior of $\Omega_{0}$.

Let $b_{n}, \bar{b}$ be the solutions of (4.1) with $k=k_{n}, \Omega=\Omega_{n}$ and $k=\bar{k}, \Omega=\Omega_{0}$, respectively. Since $\left\|g_{n}\right\|_{X}$ are uniformly bounded, (2.7) holds and Lemma 4.3 implies that $\left\|b_{n}\right\|_{C^{1, \alpha}\left(\bar{\Omega}_{n}\right)} \leq M_{1, \alpha}$, where $M_{1, \alpha}$ is independent of $n$. Therefore, there exists a subsequence $\left\{b_{n_{j}}\right\}$ and $\tilde{b} \in C^{1}(K)$ such that $\left\|b_{n_{j}}-\tilde{b}\right\|_{C^{1}(K)} \rightarrow 0$ as $j \rightarrow \infty$. Taking a sequence of compact subsets expanding to $\Omega$ and using a diagonal subsequence argument on $\left\{b_{n_{j}}\right\}$, we have $\tilde{b} \in C^{1}\left(\Omega_{0}\right)$. Let $\eta \in C_{0}^{\infty}\left(\Omega_{0}\right)$ be a test function. Then $\eta$ has compact support inside $\Omega_{n}$ for sufficiently large $n$. Hence multiplying (4.1) (a) by $\eta$ and integrating over $\Omega_{n}$, we have

$$
\int_{\Omega_{0}}\left\{-m \tilde{b}^{m-1} \nabla \tilde{b} \cdot \nabla \eta+\bar{k} \tilde{b}_{x} \eta-\tilde{b} \eta\right\}=0
$$

after taking the limit as $n \rightarrow \infty$. Hence $\tilde{b}$ is a weak solution to (4.1). The interior regularity bootstrap allows us to conclude that $\tilde{b}$ is a $C^{2}$ solution of (4.1)(a) in the interior of $\Omega_{0}$. Moreover $\|\nabla \tilde{b}\|_{L^{\infty}(\Omega)} \leq M_{1, \alpha}$.

Next we define $\tilde{b}=b_{0}$ on $\partial \Omega_{0}$ and show that $\tilde{b}$ is smooth on $\overline{\Omega_{0}}$. Let the upper half of $\partial \Omega_{n}$ be parameterized by $\mathbf{z}_{n}(t)=\left(x_{n}(t), y_{n}(t)\right), t \in[0,1]$. Likewise, let $\partial \Omega_{0}$ be parameterized by $\mathbf{z}_{0}(t)$. From our hypotheses, $\mathbf{z}_{n}(t) \rightarrow \mathbf{z}_{\mathbf{0}}(t)$ uniformly on $[0,1]$. Let $\mathbf{z}=(x, y)$ be a point in the interior of $\Omega_{0}$ and let $\mathbf{z}_{n} \in \partial \Omega_{n}, \mathbf{z}_{0}\left(t^{*}\right) \in \partial \Omega_{0}$ be such that $\left|\mathbf{z}-\mathbf{z}_{n}\right|=\operatorname{dist}\left(\mathbf{z}, \partial \Omega_{n}\right)$ and $\left|\mathbf{z}-\mathbf{z}_{0}\left(t^{*}\right)\right|=\operatorname{dist}\left(\mathbf{z}, \partial \Omega_{0}\right)$. Let $\mathbf{z}_{n}^{*} \in \partial \Omega_{n}$ be such that $\left|\mathbf{z}_{n}^{*}-\mathbf{z}_{0}\left(t^{*}\right)\right|=\operatorname{dist}\left(\mathbf{z}_{0}\left(t^{*}\right), \partial \Omega_{n}\right)$ (see Figure 2$)$. Then

$$
\left|\mathbf{z}-\mathbf{z}_{\mathbf{n}}\right| \leq\left|\mathbf{z}-\mathbf{z}_{n}^{*}\right| \leq\left|\mathbf{z}-\mathbf{z}_{0}\left(t^{*}\right)\right|+\left|\mathbf{z}_{0}\left(t^{*}\right)-\mathbf{z}_{n}^{*}\right| \leq\left|\mathbf{z}-\mathbf{z}_{0}\left(t^{*}\right)\right|+\left|\mathbf{z}_{0}\left(t^{*}\right)-\mathbf{z}_{n}\left(t^{*}\right)\right| .
$$

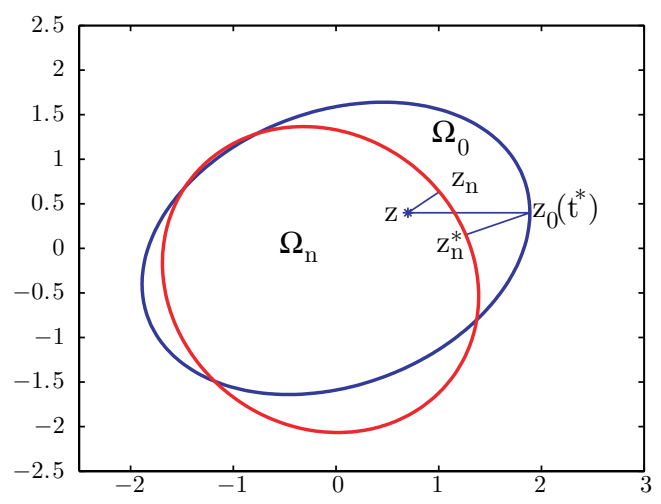

Figure 2. $\Omega_{n}$ converging to $\Omega_{0}$.

The first and last inequalities in (7.1) follow from the definition of distance from the boundary. (The point $\mathbf{z}_{n}\left(t^{*}\right)$ is not shown in Figure 2.) Since $\mathbf{z}_{n} \rightarrow \mathbf{z}_{0}$ uniformly 
on $[0,1]$, the last term in (7.1) goes to zero as $n \rightarrow \infty$. Therefore, given $\epsilon>0$, for sufficiently large $n$, we have

$$
\left|b_{0}-b_{n_{j}}(\mathbf{z})\right| \leq M_{1, \alpha} \operatorname{dist}\left(\mathbf{z}, \partial \Omega_{n}\right) \leq M_{1, \alpha}\left\{\operatorname{dist}\left(\mathbf{z}, \partial \Omega_{0}\right)+\epsilon\right\} .
$$

Letting $j \rightarrow \infty$, (7.2) is valid with $b_{n_{j}}$ replaced by $\tilde{b}$. Therefore, $\tilde{b} \in C\left(\overline{\Omega_{0}}\right)$. With $\|\nabla \tilde{b}\|_{L^{\infty}(\Omega)} \leq M_{1, \alpha}$, we can treat $\left(k \tilde{b}_{x}-\tilde{b}\right)$ as a $L^{\infty}$ source term in (4.1) with $\tilde{b}=b_{0}$ on $\partial \Omega_{0}$. Standard $L^{p}$ estimates then imply that $\tilde{b}^{m} \in W^{2, p}\left(\Omega_{0}\right)$ for any $p>1$. Together with improvement in regularity using Schauder interior estimates, we have $\tilde{b} \in C^{2, \alpha}\left(\Omega_{0}\right) \cap C^{1, \alpha}\left(\overline{\Omega_{0}}\right)$. Lemma 4.1 implies that $\tilde{b}=\bar{b}$. The proof of Step 1 is complete.

Step 2. Let $\left|\nabla b_{n_{j}}\right|(t)=\left|\nabla b_{n_{j}}\left(\mathbf{z}_{n_{j}}(t)\right)\right|$ and let $|\nabla \bar{b}|(t)=\left|\nabla \bar{b}\left(\mathbf{z}_{0}(t)\right)\right|$. Then a subsequence of $\left|\nabla b_{n_{j}}\right|(t)$ converges to $|\nabla \bar{b}|(t)$ uniformly on $[0,1]$ as $j \rightarrow \infty$.

From Lemma 4.3 and the fact that $\left|\ddot{\mathbf{z}}_{n}(t)\right|$ is uniformly bounded on $[0,1]$ (see (2.5D), $\left\{\left|\nabla b_{n_{j}}\right|\right\}$ and $\left\{\dot{\mathbf{z}}_{n_{j}}\right\}$ are uniformly Hölder continuous on $[0,1]$. Hence there exists a further subsequence of $\left\{n_{j}\right\}$, also denoted by $\left\{n_{j}\right\}$, and a function $v \in$ $C[0,1]$, such that $\left|\nabla b_{n_{j}}\right|(t) \rightarrow v(t)$ and $\dot{\mathbf{z}}_{n_{j}}(t) \rightarrow \dot{\mathbf{z}}_{0}(t)$ uniformly on $[0,1]$ as $j \rightarrow \infty$. Let $\eta \in C_{0}^{\infty}\left(\mathbf{R}^{2}\right)$, which is symmetric about the $x$-axis. Then, multiplying (4.1)(a) by $\eta$ and integrating the equation over $\Omega_{n_{j}}$, we have

$$
\begin{aligned}
0 & =\int_{\Omega_{n_{j}}}\left(\nabla \cdot\left(m b_{n_{j}}^{m-1} \nabla b_{n_{j}}\right)+k_{n_{j}} b_{n_{j} x}-b_{n_{j}}\right) \eta \\
& =m b_{0}^{m-1} \int_{\partial \Omega_{n_{j}}} \eta\left|\nabla b_{n_{j}}\right|-m \int_{\Omega_{n_{j}}} b_{n_{j}}^{m-1} \nabla b_{n_{j}} \cdot \nabla \eta+k_{n_{j}} \int_{\Omega_{n_{j}}} b_{n_{j} x} \eta-\int_{\Omega_{n_{j}}} b_{n_{j}} \eta .
\end{aligned}
$$

A similar expression holds for $\bar{b}$ and $\Omega_{0}$. Since $\left\|\nabla b_{n_{j}}\right\|_{L^{\infty}\left(\Omega_{n_{j}}\right)} \leq M_{1, \alpha}$, the result in Step 1 and the dominated convergence theorem imply that all terms involving area integrals over $\Omega_{n_{j}}$ in the second line of (7.3) go to the same integrals with $\Omega_{n_{j}}, b_{n_{j}}, k_{n_{j}}$ replaced by $\Omega_{0}, \bar{b}, \bar{k}$, respectively as $j \rightarrow \infty$. Passing to the limit and then subtracting one equation from the other, we have

$$
\int_{0}^{1} \eta\left(\mathbf{z}_{0}(t)\right) v(t)\left|\dot{\mathbf{z}}_{0}(t)\right| d t=\int_{0}^{1} \eta\left(\mathbf{z}_{0}(t)\right)\left|\nabla \bar{b}\left(\mathbf{z}_{0}(t)\right)\right|\left|\dot{\mathbf{z}}_{0}(t)\right| d t .
$$

Since $\eta$ is arbitrary, we have $v(t)=\left|\nabla \bar{b}\left(\mathbf{z}_{0}(t)\right)\right|=|\nabla \bar{b}|(t)$. The proof of Step 2 is complete.

Step 3. Let $\phi_{n}$ be the unique solution of (2.6) with $g^{*}$ replaced by $g_{n}^{*}$ and let $t_{n}(\psi)=$ $\phi_{n}^{-1}(\psi)$ for $0 \leq \psi \leq \pi$ which is defined since $d \phi_{n} / d t>0$. Let $\bar{t}(\psi)=\bar{\phi}^{-1}(\psi)$ be similarly defined. Then there exists a subsequence of $\left\{n_{j}\right\}$ which is defined in Step 2 , denoted the same, such that $t_{n_{j}}$ converges to $\bar{t}$ in $C^{1}[0, \pi]$.

Since $d \bar{\phi} / d t$ is positive and continuous on $[0,1]$, there exist positive constants $\delta$ and $M$ such that $\delta<d \bar{\phi} / d t<M$ on $[0,1]$. From Remark 4 in $\S 3$, there exists a subsequence of $\left\{n_{j}\right\}$, denoted the same, such that $\phi_{n_{j}} \rightarrow \bar{\phi}$ in $C^{1}[0,1]$. Therefore, $\delta<d \phi_{n_{j}} / d t<M$ on $[0,1]$ for sufficiently large $j$. Differentiating $\phi_{n}^{-1}\left(\phi_{n}(t)\right)=t$, we have $1 / M \leq d \phi_{n}^{-1} / d \psi \leq 1 / \delta$ on $[0, \pi]$. Therefore, there exist a $v \in C[0, \pi]$ and a further subsequence such that $\phi_{n_{j}}^{-1} \rightarrow v$ in $X$. Using the fact that $\phi_{n_{j}} \rightarrow \bar{\phi}$ in 
$C[0,1]$, it is easy to see that $v=\bar{\phi}^{-1}$ so that $t_{n_{j}}=\phi_{n_{j}}^{-1} \rightarrow \bar{t}=\bar{\phi}^{-1}$ in $X$ as $j \rightarrow \infty$. The proof of Step 3 is complete.

To finish the proof, recall that $T_{2}\left(k_{n}, \Omega_{n}\right)=\left|\nabla b_{n}\right|\left(t_{n}(\psi)\right)$ and $T_{2}(\bar{k}, \bar{\Omega})=$ $|\nabla \bar{b}|(\bar{t}(\psi))$. Step 2 shows that $\left|\nabla b_{n_{j}}\right|(t) \rightarrow|\nabla \bar{b}|(t)$ uniformly on $[0,1]$, and Step 3 shows that $t_{n_{j}}(\psi) \rightarrow \bar{t}(\psi)$ uniformly on $[0, \pi]$. Therefore, $T_{2}\left(k_{n_{j}}, \Omega_{n_{j}}\right) \rightarrow T_{2}(\bar{k}, \bar{\Omega})$ in $C[0, \pi]$. The proof of Lemma 4.4 is complete.

\section{REFERENCES}

[1] Amann, H. and M. G. Crandall (1978). On some existence theorems for semi-linear elliptic equations. Indiana U. Math. J. 27, 779-790. MR503713 (80a:35047)

[2] Berestycki, Henri and Louis Nirenberg (1992). Traveling fronts in cylinders. Ann. Inst. H. Poincaré Anal. Non Linéaire 9, 497-572. MR.1191008 (93k:35019)

[3] Brazhnik, Pavel and John Tyson (2000). On traveling wave solutions of Fisher's equation in two spatial dimensions. SIAM J. Appl. Math. 60, 371-391. MR.1740251 (2001a:35088)

[4] Choi, Y.S., Juliet Lee and Roger Lui (2004). Traveling wave solutions for a one-dimensional crawling nematode sperm cell model. J. Math. Bio. 49, 310-328. MR2102761 (2005g:92011)

[5] Choi, Y.S. and Roger Lui (2004). Existence of traveling wave solutions for a one-dimensional cell motility model. Taiwanese Journal of Mathematics, 8, 399-414. MR2163314|(2006c:35030)

[6] Choi, Y.S., Patrick Groulx and Roger Lui (2005). Moving boundary problem for a onedimensional crawling nematode sperm cell model, Nonlinear Analysis: Real World Applications 6, 874-898. MR2165218 (2006f:35283)

[7] Choi, Y.S. and Roger Lui. Real eigenvalues of a linearized operator arising a moving boundary problem. Proceedings of AMS (accepted).

[8] Evans, Lawrence (1998). Partial Differential Equations, Graduate Studies in Math., 19, Amer. Math. Soc. MR1625845 (99e:35001)

[9] Fife, Paul (1979). Mathematical Aspects of Reacting and Diffusing Systems, Lecture Notes in Biomath, vol. 28, Springer, NY. MR527914 (80g:35001)

[10] Gilbarg, David and Neil Trudinger (1998). Elliptic Partial Differential Equations of Second Order. Springer, New York.

[11] Mogilner, A. and D. W. Verzi (2003). A simple 1-D physical model for the crawling nematode sperm cell. J. Stat. Phys. 110, 1169-1189.

[12] Pelcé, Pierre (1988). Dynamics of Curved Fronts. Academic Press, Boston. MR986791 (91b:76002)

[13] Su, Jianzhong (2001). On the existence of finger solutions of the Hele-Shaw equations. Nonlinearity 14, 153-166. MR:1808629 (2001m:76039)

[14] Volpert, Vitaly A. and Valdimir Volpert (1994). Traveling Wave Solutions of Parabolic Systems, Translations of Mathematical Monographs, 140, Amer. Math. Soc. MR 1297766 (96c:35092)

Department of Mathematics, University of Connecticut, Storrs, Connecticut 06269

E-mail address: choi@math.uconn.edu

Department of Mathematical Sciences, Worcester Polytech Institute, Worcester, MASSACHUSETTS 01609

E-mail address: rlui@wpi.edu 